\title{
A rare, but to be considered disease, in the differential diagnosis of
}

\section{abdominal pain: Mesenteric Panniculitis}

\author{
Bülent Yardımcı ${ }^{1} *$
}

\begin{abstract}
Abdominal pains are one of the major topics of emergency clinics. Mesenteric panniculitis (MP) is one of the rarely encountered causes of abdominal pain and is known as an idiopathic disease with a good prognosis. MP may cause abdominal pain, abdominal mass and intestinal obstruction. Infections, autoimmune diseases and abdominal traumas may trigger MP. The disease may coexist with malignancy and has a high probability of developing malignancy in MP patients. In this case report, a 59-year-old female patient, who came to our Internal Medicine Outpatient Clinic with abdominal pain and who was diagnosed with MP, is presented.
\end{abstract}

Keywords: Mesenteric Panniculitis, Mesenteric Weber-Christian disease, Abdominal pain, Mesenteric lipodystrophy

\section{Introduction}

Mesenteric panniculitis (MP) is a non-specific inflammatory disease of the mesenteric fatty tissue (1). Its prevalence was reported to be at a rate of $0.16-3.3 \%$ (2). It is mostly seen in men and the ratio of men to women is 2$3 / 1$. The disease is frequently seen in patients at an age of about 50-60 years. The clinical picture varies widely, from incidentally caught asymptomatic ones to cases with mass(es) detected in the abdomen (3). Initially, it was defined by Jura in 1924 and is known by the names mesenteric lipodystrophy, retractile mesenteritis, liposclerotic mesenteritis, or mesenteric Weber-Christian disease (4).

\section{Material and method}

A 59-year-old female patient presented to our Internal Medicine Outpatient Clinic with blunt abdominal pain with pain scale severity between 6 and 7. It had been happening for three days and the severity of the pain was gradually increasing, comprising the upper quadrants and the periumbilical area, and with a dull feeling of nausea. She denied having any other complaints, such as lack of appetite, fatigue, vomiting, fever, diarrhea, constipation, or distention.

The patient, who stated that she was defecating regularly every day, did not have any urinary symptoms. She had been diagnosed with Hashimoto thyroiditis, hypertension, hypercholesterolemia, and psoriatic arthritis in her history and she had undergone cholecystectomy 6 years ago.
The patient stated that she had given a normal, healthy birth 25 years ago and that she has not undergone any gynecological disease.

There was no history of alcohol consumption and smoking. There was no severe disease in her family history. She was on the following medications: Levothyroxine $100 \mathrm{mg} \mathrm{qd}$, amlodipine $10 \mathrm{mg}$ qd, nebivolol $5 \mathrm{mg}$ qd, atorvastatin 10 $\mathrm{mg} 1 \mathrm{x} 1$, methotrexate $10 \mathrm{mg}$ per week.

On physical examination, blood pressure was 110/70 mm $\mathrm{Hg}$, heart rate was $84 / \mathrm{min}$ and fever was $37.2{ }^{\circ} \mathrm{C}$. The epigastrium and umbilical area were sensitive on abdominal examination. However, there was no defense or rebound and bowel sounds were hyperactive. There were no pathological findings.

As a first step blood and urine tests were done. The patients laboratory test results are shown in Table 1. Urinalysis was normal.

The abdominal computed tomography (CT) scan showed hypodense nodules (cyst) in the liver, the largest of which was measured $7 \mathrm{~mm}$.

CT findings showed the mesenteric fatty planes with paramedian localization at the abdominal midline showing signs of panniculitis, and atherosclerotic changes were determined at the main vascular structures (Image 1). 
Table 1: Laboratory findings of the Patient

\begin{tabular}{lll} 
& Patient's results & Normal range \\
\hline ALT & $25 \mathrm{U} / \mathrm{L}$ & $20-40 \mathrm{U} / \mathrm{L}$ \\
AST & $17 \mathrm{U} / \mathrm{L}$ & $20-40 \mathrm{U} / \mathrm{L}$ \\
GGT & $18 \mathrm{U} / \mathrm{L}$ & $0-45 \mathrm{U} / \mathrm{L}$ \\
Pancreatic Amylase & $24 \mathrm{U} / \mathrm{L}$ & $20-80 \mathrm{U} / \mathrm{L}$ \\
Lipase & $31 \mathrm{U} / \mathrm{L}$ & $10-140 \mathrm{U} / \mathrm{L}$ \\
C-Reactive Protein & $8.4 \mathrm{mg} / \mathrm{L}$ & $0,5 \mathrm{mg} / \mathrm{L}$ \\
Leucocyte & $4.47 \mathrm{x} 10^{\wedge} 3 / \mathrm{uL}$ & $4,1-11,1 \times 10^{\wedge} 3 / \mathrm{uL}$ \\
Haemoglobin & $13.1 \mathrm{~g} / \mathrm{dL}$ & $11,7-15,5 \mathrm{~g} / \mathrm{dL}$ \\
Haematocrit & $39 \%$ & $35-45 \%$ \\
Thrombocyte: & $240,000 /$ microliter & $150,000-400,000 /$ microliter \\
\hline
\end{tabular}

U/L:unit/liter , mg/L:milligram/liter , g/L:gram/liter , g/dL:gram/deciliter, Alanine Aminotransferase (ALT), Aspartate Aminotransferase: (AST), Gamma Glutamyl Transferase (GGT)

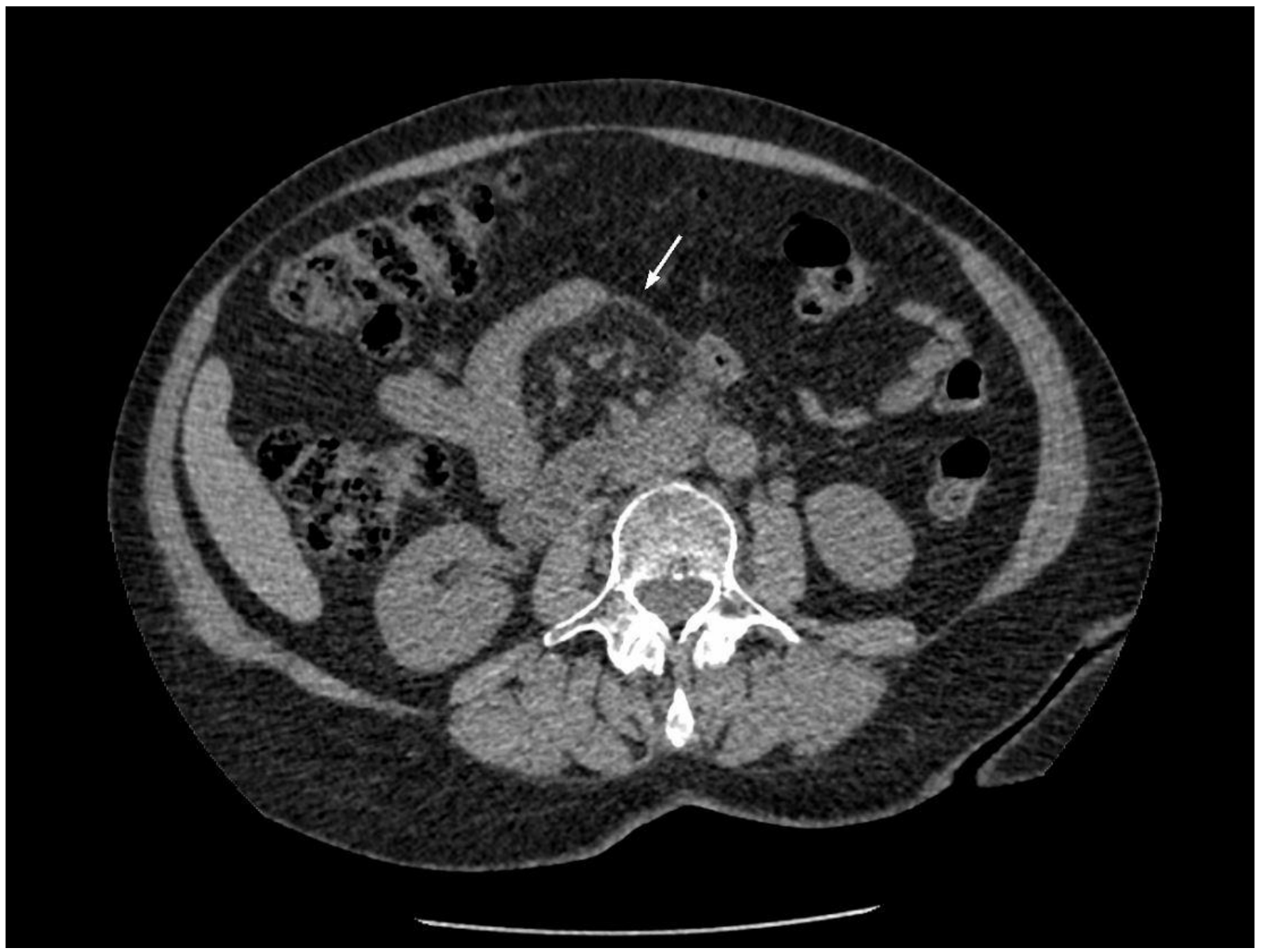

Figure 1: View of Mesenteric Panniculitis in Patient's Computed Tomography. (Arrow shows 'Fat Ring Sign')

\section{Discussion}

Anatomically, the mesenterium is a structure extending from the duodenojejunal flexura to the mesorectum. MP is an IgG4-related auto-immune disease progressing with the inflammation of the fatty tissue (5).

The pathogenetic mechanism of the disease could not be fully understood. However, it is observed together with an agent that it is triggering non-specific inflammation in the adipose tissue. These triggers may be rheumatoid and autoimmune diseases such as primary sclerosing cholangitis, rheumatoid arthritis, Sjogren's syndrome.
Other causes are infections such as typhoid fever, tuberculosis; peptic ulcer, gallbladder stone, abdominal trauma, abdominal surgery, and malignancies such as lymphoma and mesenteric tumors. Malignant diseases which cause MP most commonly are breast cancer, nonHodgkin lymphoma, colon cancer, and lung cancer $(4,5)$.

The findings of the patients are variable. The patients may be asymptomatic or may show findings such as abdominal pain, nausea, vomiting, constipation, diarrhea, intestinal obstruction and palpable abdominal mass. 
The findings of the disease may present within a few days or may progress as a chronic disease for several years. In different clinical series, abdominal pain $(70 \%)$, distention and gas $(26 \%)$, and diarrhea $(25 \%)$ are the most common symptoms and $10-27 \%$ of the patients were reported to be asymptomatic (1).

In our case, the main symptom was an abdominal pain and slight nausea. The patient had auto-immune diseases, such as Hashimoto thyroiditis and psoriatic arthritis. In previous cases, auto-immune diseases such as Riedel thyroiditis, Sjögren's syndrome, and rheumatoid arthritis was reported to coexist with the disease. In the laboratory tests, the CReactive Protein (CRP) result was slightly higher than its normal value. Thereforer, we can differentiate her condition from a severe acute abdomen. The most common positive laboratory finding observed in MP is high sedimentation rate and a high CRP (4).

For the diagnosis of the disease, ultrasonography is generally not sufficient. Thickening of the mesenteric root and a slight decrease in echogenicity may be determined. The diagnosis is generally established by means of CT. Mesenteric involvement is its typical symptom and further signs of MP are an increase in the density of the mesentery with intercalated nodules, a "fat-ring sign" around vessels in the form of a halo, the formation of a pseudocapsule and the displacement of bowel loops. Magnetic resonance imaging (MRI) findings are similar to those of CT (6). In our case, the diagnosis was reached by CT. The patient's pain declined with symptomatic therapy. Although the pain in this disease responds well to corticosteroid treatment in a short time, this was not necessary for our patient. Apart from the treatment of the accompanying diseases, surgical intervention may be necessary in cases of obstruction. However, even after surgical interventions, there is a probability of recurrence of panniculitis (5).

In MP, it is important to exclude malignancy. Along with the suggestions to handle MP in patients like a paraneoplastic syndrome, there are also publications asserting that the probability of the development of malignancy increases about 5 times (7).

Differential diagnosis is very important. Since the presenting symptoms of the disease are not specific, it can be misdiagnosed. MP may present itself with intestinal obstruction or abdominal mass. If the patient is misdiagnosed, it may result in a surgical operation. Although rarely, in some MP patients presented with intestinal obstruction ; a surgery intervention is necessary. But it should also be noted that only a small portion of these patients needed no additional treatment (8).

Therefore, after the regression of the patient's acute condition, tests were planned in order to investigate underlying diseases or malignancies. No pathological finding was observed on autoimmune markers, gynecologic tests, mammography, positron emission tomography (PET) scan, gastroscopic and colonoscopic examinations.

\section{Conclusion}

Although MP is generally a chronic mesenteric inflammation, the patients may present with an acute condition such as abdominal pain, nausea, vomiting, diarrhea, abdominal mass, and bowel obstruction. Since it is a rarely encountered disease, it may initially not come to mind in the differential diagnoses. When there is no guiding symptom in the abdominal ultrasonography, which is the method of analysis most frequently used by us at the emergency station as first step, it should be kept in mind that in the evaluation of these patients CT is especially of significance and CT scan should be made as soon as possible for the necessary cases.

\section{Acknowlegment: None}

Author Contributions: BY: Review of the literature, Project design, Patient examination, data collection and analyzes BY: Writing and Revisions

Conflict of interest: No actual or potential conflicts of interest exist in relation to this article.

Ethical issues: All authors declare originality and ethical approval of research. Responsibilities of research, responsibilities against local ethics commission are under the authors responsibilities. The study was conducted under defined rules by the local ethics commission guidelines and audits.

\section{References}

1. Buragina G, Biasina AM, Carrafiello. Clinical and radiological features of mesenteric panniculitis: a critical overview. Acta Biomed. 2019 Dec 23;90(4):411-422

2. Protin-Catteau L, Thiéfin G, Barbe C, Jolly D, Soyer P, Hoeffel C. Mesenteric panniculitis: review of consecutive abdominal MDCT examinations with a matched-pair analysis. Acta Radiol. 2016 Dec;57(12):1438-1444

3. Kniazkova I, Korchevskaya A, Bogun M. Clinical case of mesenteric panniculitis. Reumatologia 2019; 57, 5: 297-300

4. Mehta P, Reddivari AKR, Ahmad MA. Case Report of Mesenteric Panniculitis.2020, [Cited 2020 March 12] Avaliable from URL https://www.cureus.com/articles/26936-a-case-report-of-mesentericpanniculitis/metrics

5. Gogebakan O, Osterholf AM, Albrecht T. Mesenteric Panniculitis (MP): A Frequent Coincidental CT Finding of Debatable Clinical Significance. Fortschr Röntgenstr 2018; 190: 1044-1052

6. Morgan MA, Singh G, et al. Sclerosing mesenteritis [Cited 2020 March 12] Avaliable from URL https://radiopaedia.org/articles/sclerosing-mesenteritis-1

7. Scheer F, Spunar P, Wiggermann P, Wisgoft C, Andresen R. Mesenteric Panniculitis (MP) in CT - A Predictor of Malignancy? Fortschr Röntgenstr 2016; 188: 926-932

8. Akram S, Pardi DS, Schaffner JA, Smyrk TC: Sclerosing mesenteritis: clinical features, treatment, and outcome in ninety-two patients. Clin Gastroenterol Hepatol. 2003, 10:103-111.

Copyright (C) 2020 The Author(s); This is an open-access article distributed under the terms of the Creative Commons Attribution License (http://creativecommons.org/licenses/by/4.0), (CC BY NC) which permits unrestricted use, distribution, and reproduction in any medium, provided the original work is properly cited. International journal of Medical Science and Discovery. 